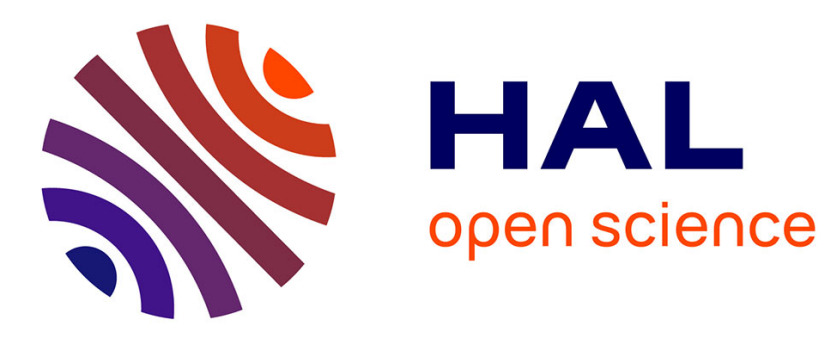

\title{
Using the fractal Code to Watermark Images
}

Patrick Bas, J.-M. Chassery, F. Davoine

\section{- To cite this version:}

Patrick Bas, J.-M. Chassery, F. Davoine. Using the fractal Code to Watermark Images. ICIP, Aug 1998, France. pp.469-473. hal-00166548

\section{HAL Id: hal-00166548 \\ https://hal.science/hal-00166548}

Submitted on 7 Aug 2007

HAL is a multi-disciplinary open access archive for the deposit and dissemination of scientific research documents, whether they are published or not. The documents may come from teaching and research institutions in France or abroad, or from public or private research centers.
L'archive ouverte pluridisciplinaire HAL, est destinée au dépôt et à la diffusion de documents scientifiques de niveau recherche, publiés ou non, émanant des établissements d'enseignement et de recherche français ou étrangers, des laboratoires publics ou privés. 


\section{Using the fractal code to watermark images}

\author{
Patrick Bas, Jean-Marc Chassery \\ UMR CNRS TIMC \\ I.A.B. \\ Domaine de la merci \\ 38706 La Tronche cedex, France \\ Patrick.Bas@imag.fr \\ Jean-Marc.Chassery@imag.fr
}

\author{
Franck Davoine \\ UMR CNRS Heudiasyc \\ U.T.C. \\ BP 20529 \\ 60205 Compiegne cedex, France \\ Franck.Davoine@hds.utc.fr
}

\begin{abstract}
Our paper present a watermarking scheme basing on an insertion of similarities. In a first part different watermarking techniques are presented and classed. In a second part our scheme is described in its spatial and frequential implantations. Finally the different results and perspectives of the work are outlined.
\end{abstract}

\section{INTRODUCTION}

With the democratization of the multimedia technology, it is possible to get original numerical images and to appropriate it. To prevent this problem, engineers wanted to add copyright on numerical work. This leads to watermarking.

The watermarking consists in hiding a binary mark which is indelible and robust to image processing techniques such as cropping, blurring, to geometrical transformations, or even lossy compression techniques.

\subsection{Previous Work}

The watermarking schemes can be divided into two categories: a first uses the spatial domain. It consists in adding a tag on the original image. This mark is characterized by its geometric or statistic properties. The least significant bits can be altered by a M-sequence [1]. A particular pattern can be added in the image [2]. A second category of scheme uses the frequency domain. A domain such as the DCT one permits the mark to be less visible and more robust to compression techniques. Zhao uses the DCT on $8 \times 8$ DCT blocks and altered specific coefficients [3]. Cox developed a spread-spectrum based scheme to embedded a mark [4]. Techniques benefit from the visual properties of the eye to mask the frequential components inherent to the watermark inside the image [5].

\section{$1.2 \quad$ Different applications}

It exists different ways of embedding and detecting a mark in an image. We can distinct four classes:

- the detection step uses the non-marked image [4]: only a reduced group of person can detect the mark

- the detection step does not use the non-marked image. Every-body can detect the presence of a mark

- the embedding and detecting schemes are known: the scheme needs a key to prevent a pirate from removing the mark

- the embedding and detecting schemes are not known: the schemes are secret but can be cracked [6]

This diversity of schemes leads to different watermarking protocols and performances.

\section{WATERMARKING USING THE COLLAGE MAP}

\subsection{The Collage Map}

Our approach used the fractal code developed by Jacquin [7] in fractal compression. This fractal code, which can be seen as a collage map, extracts the selfsimilarities of the image. It is generated by calculating an Iterated Function System (IFS) from the image. The image is partitioned into two kinds of blocks: the 
range and the domain blocks that are respectively extracted from a range partition $R$ and a domain partition $D$. The Collage Map is built by associating to each block $R_{i}$ of the partition $R$, the block $D_{j}$ which is more similar to $R_{i}$ (except itself). This test of selfsimilarity consists in finding the couple of reals $s$ and $o$ minimizing a quadratic error $e$ between the block $R_{i}$ and the affine transformed block $D_{j}=s * R_{i}+o[7]$. To each image corresponds a Collage Map composed of a range partition $R$, the indices $I_{j}$ of associated blocks of partition $D$, the scale $s_{j}$ and the offset $o_{j}$ (cf fig 1). It can be written as follows:

Collage $\operatorname{Map}=\left\{R, I_{1}, \ldots, I_{n}, s_{1}, \ldots, s_{n}, o_{1}, \ldots, o_{n}\right\}$

This map representing similarities in the image can also be considered in the frequential domain, it is used for fractal image compression by Barthel [8].

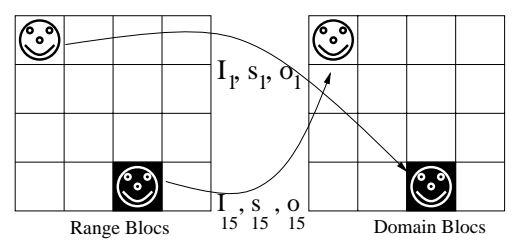

Figure 1: principle of a Collage Map

\subsection{Adding the mark}

The mark is embedded altering the original Collage Map. Because it is statistically rare to find a block similar to another in an ordinary image (except when the image is a fractal image), adding similarities permits to obtain singular information in the image. Our algorithm adds artificial and visually invisible local similarities into the image in order to control the Collage Map. This is done by substituting a Range block $R$ with a new block $\hat{R}=s . D+o$. By this way we force new exact mappings instead of the default best original mappings. By adding exact domain similarities in the image (that define the watermark), we control the Collage Map.

To avoid block artifact and to improve the watermark invisibility, we also perform the embedding scheme in the DCT domain.

Two problems have to be solved: which blocks will be candidate and how to perform the positioning of the similarities.

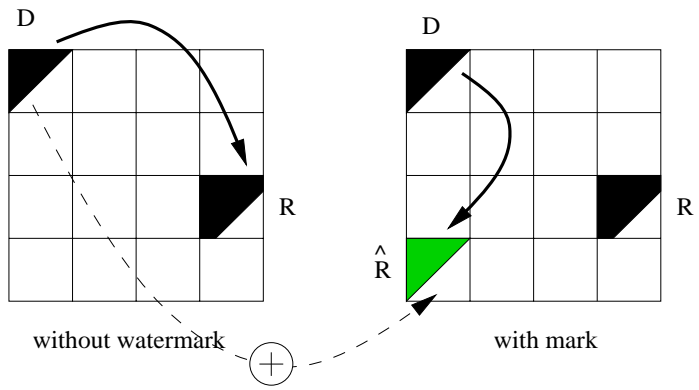

Figure 2: principle of embedding (collages are represented by solid lines)

\subsection{Domain blocks and Range blocks se- lection}

The structure of a natural image is very complex. There are homogeneous areas, noisy/textured areas, and edge areas . To be robust to the different compressing methods, marking must have a low-frequency component which is significant. The selection of the Domain blocs must also be robust to low-pass filtering (i.e. compression schemes) and geometric transformations as rotation or translation.

We select these interest blocks by calculating a criterion like their standard-deviation (for the spatial scheme) or their low-frequency component (for the DCT-based scheme)(fig 3.b).

To ensure an efficient detection step, one Domain

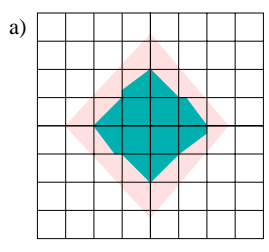

Partitionned Image

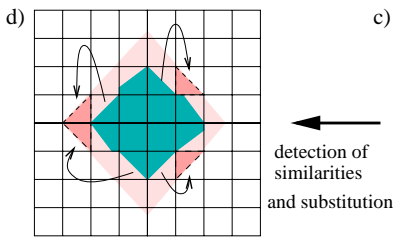

Figure 3: The embedding stage block must not be similar to another. Consequently, we proceed to a block quantization of the Domain pool (fig 3.c). This can be done by classing blocks with similar criterion.

The Range blocks $R$ are selected to be similar with 
$\hat{R}=s . D+o$ for specific real values $s$ and $o$. Therefore the information added will be as invisible as possible (cf figure 3 ). The distance between two blocs is calculated using the quadratic error (fig 3.d).

\subsection{Spatial domain embedding}

The image is partitioned in blocks of $8 \times 8$ pixels size. The dynamic of each $\hat{R}$ block is chosen less than 20.0. Otherwise block artifacts will appear and the similarities are perceptible.

According to the image and the hiding quality, the number of Domain Blocks is between 50 to 100 .

The magnitude of the watermark is fixed by a factor $S$. For each blocks $D$ and $R, \hat{R}$ is calculated as follows:

$$
\hat{R}=\delta * S * \frac{D}{\max (D)}+\bar{R}
$$

where $\bar{R}$ is the mean of $R$ and

$$
\delta=\left\{\begin{array}{cl}
+1 & \text { if the embedded bit }=1 \\
-1 & \text { if the embedded bit }=0
\end{array}\right.
$$

The quadratic error between $R$ and $\hat{R}$ is then calculated.

\subsection{DCT domain embedding}

The $8 \times 8$ blocks of the image are transformed to DCT coefficients and we consider the similarity between only the low frequency coefficients (cf figure 4). Thus the higher frequency coefficients permit to mask the watermark and moreover the low frequency coefficients are less altered by compression techniques.

In this case, $\hat{R}$ is given by:

$$
\hat{R}=R_{h p}+\delta * S * \frac{D_{l p}}{\max \left(D_{l p}\right)}
$$

where.$h p$ and.$l p$ are the high-pass components (plus the DC coefficient) and low-pass components.

$$
\delta= \begin{cases}+1 & \text { if the embedded bit }=1 \\ -1 & \text { if the embedded bit }=0\end{cases}
$$

To obtain invisibility of the watermark, maximum DCT coefficients magnitudes must be smaller than 200.0. The similarities are searched in the luminance space to avoid quantization problems after the inverse DCT transform.

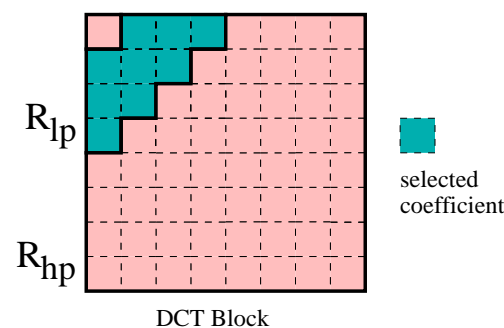

Figure 4: DCT block and modified coefficients

\subsection{Watermark detection step}

The detection can be applied to prove the existence of the watermark and to read it. To perform this, the identifier needs the location of the Range Blocks. Let $p_{1}$ be a counter that express the number of matched blocks. Our detection scheme consists in :

1. Get a Domain block $D$ of the image

2. Create a block $\hat{R}$ (cf formula 1 and 2 ) and search the Range block $R$ which minimizes the quadratic error.

- If the index $R$ is the same that the index in the table, $p_{1}$ is increased and the embedded bit is deduced from the sign of $\delta$.

- If the index of $R$ is not the same than the index in the table, $p_{1}$ does not change.

3. Get another Domain block $D$ of the image and go to 2 until you have no more block $D$.

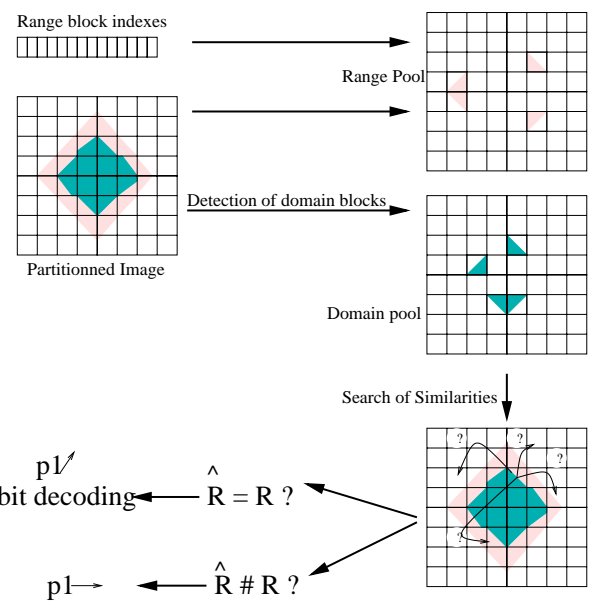

Figure 5: Detection Stage 


\subsection{Results and Perspectives}

\subsubsection{Results}

The "spatial" and "frequential" algorithms have been tested on lena $256 \times 256$. For each scheme, 50 Domain blocks have been selected. The number of Range blocks detected (and the number of decoded bits) is calculated for different quality factors with the JPEG compression scheme (cf figure 6 and 7 ).

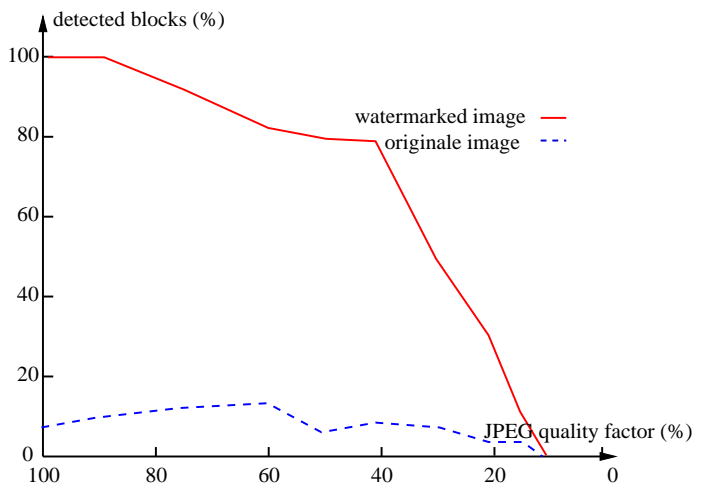

Figure 6: JPEG-test with the DCT scheme

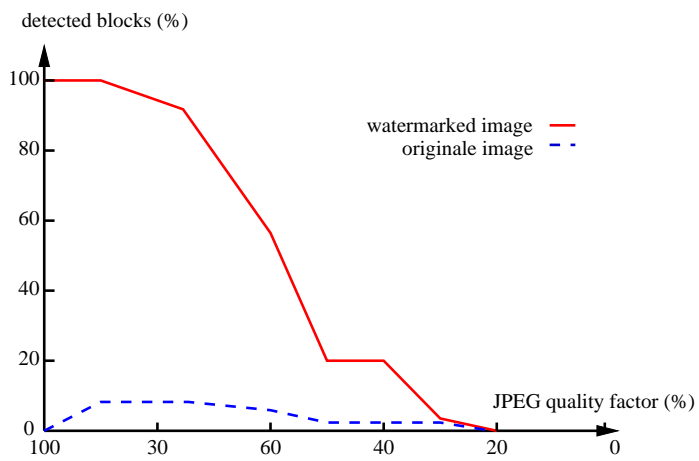

Figure 7: JPEG-test with the spatial scheme

For a same distortion ( $P S N R=52 d B$ ) we can notice that the DCT scheme is more robust to the JPEG test (more blocks are detected). This is due to the low-pass embedding of the DCT method. Furthermore this method does not produce disturbing block artifacts as the spatial method does.

The number of Domain blocks is limited by the dynamic of the image. If the image does not contain edges or contains low-dynamic edges the detection step is less robust to compression techniques.
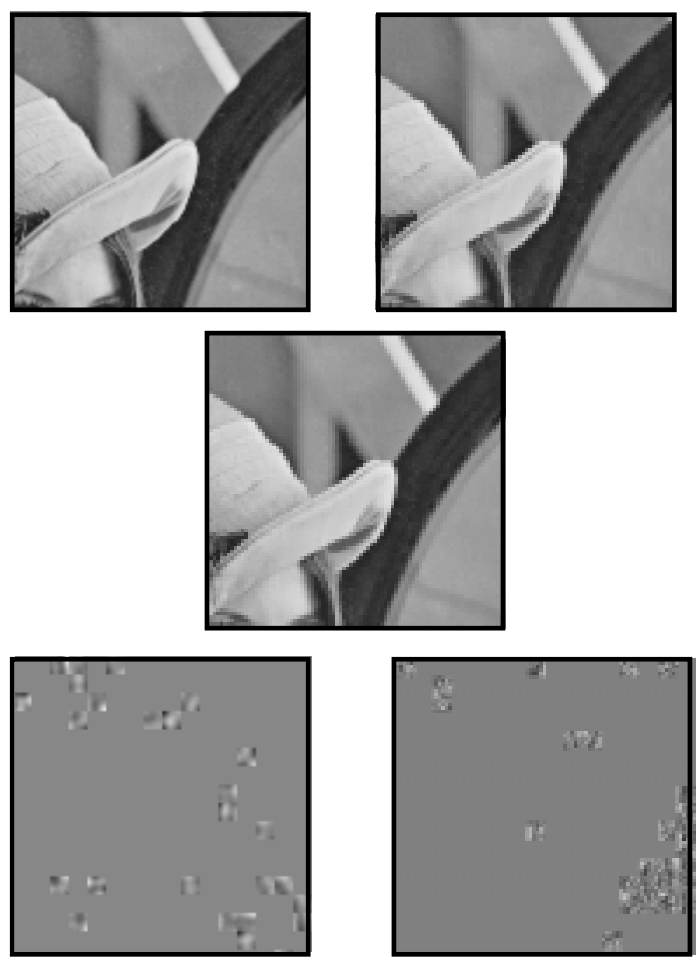

Figure 8: In the left part: the watermarked image and the added information (rescaled) using our DCT algorithm, in the right part: the watermarked image and the added information (rescaled) using our spatial algorithm, in the middle part: the original image

\subsubsection{Perspectives and Future Work}

At this time, our scheme uses a key given by indexes of the range blocks. Many improvements are planed to be implemented during the next months:

1. a local insertion of the similarities to be robust to cropping

2. an adaptative insertion of the DCT similarities

3. the presence of a key which permits to build a Range blocks pool

The first point can be implanted by reducing the search domain(cf fig9.1). The second point could be easily done by preserving the highest components of the Domain block. By doing this, the masking process will be improved (cf fig9.2, fig10). The third point uses a key which generated a list of Range blocs and defines a Range pool. Consequently if an user wants to extract the mark, he needs the key(cf fig9.3). We also plan to add a "universal" but less robust insertion which permits every-body to get basic information from the image (i.e. an identification number). 

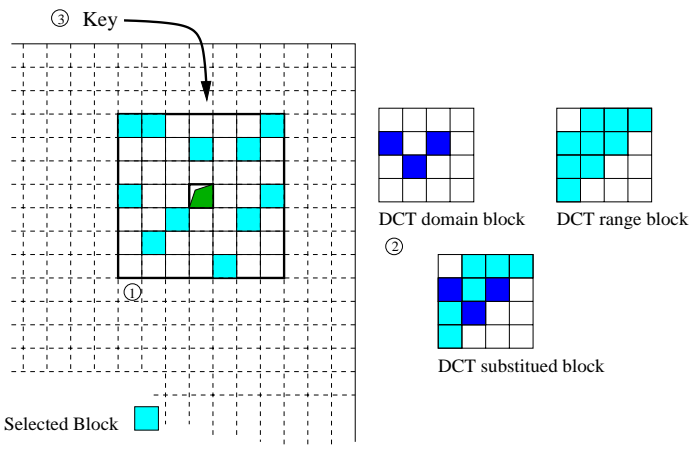

Figure 9: Perspectives: local search (1), adaptative insertion (2), key(3)

\section{CONCLUSION}

Our work presented a watermaking scheme using similarities to embed a mark. We have developed two different algorithms: the first adds similarities in the spatial domain, the second in the DCT domain. Our studies indicates that the DCT based scheme is more robust to compression than the spatial one. The general framework permits to foresee many perspectives as the enhancement of the masking part and the use of a key.

\section{References}

[1] R.B. Wolfgang and E.J. Delp. A watermarking technique for digital imagery: Further studies. In Int. Conf. on Imaging Science, Systems and Technology, Las Vegas, Nevada, Juillet 1997.

[2] G. Caronni. Assuming ownership rights for digital images. In Reliable IT System, VIS'95, 1995.

[3] Koch E. Zhao J. Embedding robust labels into images for copyright protection. Technical report, Fraunhofer Institute for Computer Graphics, Darmstadt, Germany, 1994.

[4] T. Leighton I. Cox, J. Killian and T. Shamoon. Secure spread spectrum watermarking for multimedia. IEEE Transactions on Image Processing, 6(12):1673-1687, December 1997.

[5] Goffin F., Delaigle J.-F., De Vleeschouwer Ch., Macq B., and Quisquater J.-J. A low cost perceptive digital picture watermarking method. In Proceedings of the SPIE: Electronic Imaging, volume SPIE Vol. 3016, February 1997.

[6] Fabien A.P. Petitcolas, Ross J. Anderson, and Markus G. Kuhn. Attacks on copyright mark-

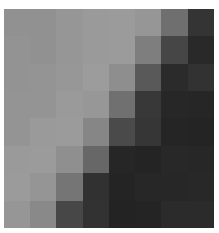

Range block err $=13887.34$

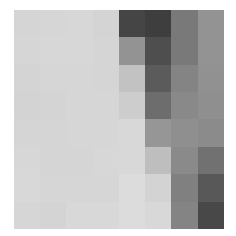

Domain block

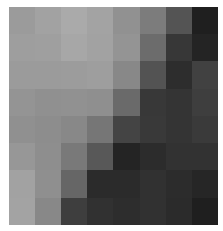

DCT similar block:

err $=1.88$

Figure 10: example of adaptative DCT substitution: the 5 highest components of the Range block are preserved, the other (10) are preserved, the collage error between the Range block and the Domain block is calculated.

ing systems. In David Aucsmith, editor, Information hiding: second international workshop, Lecture notes in computer science. Springer Verlag, Berlin, Germany, 1998.

[7] A. E. Jacquin. Image coding based on a fractal theory of iterated contractive image transformations. IEEE Transactions on Image Processing, 1(1):18-30, January 1992.

[8] K. U. Barthel and T. Voyé. Adaptive fractal image coding in the frequency domain. In Proceedings of International Workshop on Image Processing: Theory, Methodology, Systems and Applications, Budapest, Hungary, June 1994. 